\title{
Multi-Perspective High Range Resolution Profiles of Landmines
}

\author{
Lauren Wright and Alessio Balleri \\ Centre for Electronic Warfare \\ Cranfield University \\ Defence Academy of the UK \\ Shrivenham, UK \\ a.balleri@cranfield.ac.uk
}

\author{
Hugh Griffiths and Federico Lombardi \\ Dept of Electronic \& Electrical Eng. \\ University College London \\ London, UK \\ h.griffiths@ucl.ac.uk
}

\begin{abstract}
Landmine clearance is a severe and unresolved humanitarian issue. The development of low-cost, smaller, faster and lighter Ground Penetrating Radars (GPR), which can be mounted on unmanned platforms, will allow faster and safer 24/7 operations. This technology will make it possible to survey affected areas with more flexible trajectories and these will provide measurements of landmine signatures from many different aspect angles. As a result, multi-perspective information over wide angular windows and the behaviour of the signature as a function of the angle of illumination can be exploited. Landmine signatures are expected to present features that are less sensitive to the angle of illumination with respect to those of common cluttered objects, and this can lead to an improvement in detection and discrimination performance.

In this paper, we present the results of an experimental trial carried out to collect the High Range Resolution Profiles (HRRPs) of two landmines, the SB-33 and the VS-50, off the ground. An analysis of the auto-correlation function of each range bin as a function of the aspect angle is presented together with that of the cross-correlation between profiles collected from different aspect angles.
\end{abstract}

\section{INTRODUCTION}

Anti-personnel landmines have been used extensively as an indiscriminate weapon since World War II both by professional military forces and in militia/insurgent operations. In recent years, the impact of landmines that have remained in wartorn countries after the end of a conflict has drawn significant attention, and it is recognised as a severe and unresolved humanitarian issue. The populations of countries that are affected by landmines are regularly killed and maimed. In addition to this, the resulting social and medical costs that these countries are facing burden and retard their economical recovery.

The Landmine Monitor is an excellent source of information describing this problem [1]. According to the Landmine Monitor, in 2013 a global total of 3,308 casualties from landmines and Explosive Remnant of War (EWR) were recorded. This corresponds to an incidence rate of 9 casualties per day. Of these, at least 1,065 were killed. The $79 \%$ of casualties were civilians and $46 \%$ were children. In $2013,49 \%$ of all casualties for which the specific type of victim-activated explosive item was known were caused by anti-personnel mines or victim-activated IEDs acting as anti-personnel mines. In many states and areas, numerous casualties remain unrecorded and therefore the true number of causalities is anticipated to be significantly higher. As of October 2014, 56 states and 4 additional areas still remain threatened from landmines. It is difficult to have an estimate of the total buried number of landmines but it is believed that four countries are still massively contaminated (over $100 \mathrm{~km}^{2}$ ), that at least five are heavily contaminated (between $20 \mathrm{~km}^{2}$ and $100 \mathrm{~km}^{2}$ ) and that the remaining countries are at medium $\left(3 \mathrm{~km}^{2}\right.$ to $\left.20 \mathrm{~km}^{2}\right)$ or light contamination (less than $3 \mathrm{~km}^{2}$ ). Removing landmines without detonation costs from 300 USD to 1000 USD per landmine. It is estimated that to clear these areas using current technologies would cost an estimated 30 billion USD and would take over 1000 years [2].

Currently, there is no single answer to the problem of landmine detection and localisation. Various techniques have been proposed in the past, such as gravity and magnetic surveying, seismic and nucleonic, but none of these have yet offered a complete solution to the problem. Ground Penetrating Radar (GPR) has proved to be between the most relatively successful techniques and it is still a very attractive option on which much is being published. An excellent source of information on GPR is [3].

Most of previous research on landmine detection and localisation has focussed on improving the performance of existing GPR systems [4][5]. These include methods that exploit polarimetric differences in GPRs [6][7]. A new technique based on Hidden Markov Models (HMM) and Multiple-Instance Learning (MII) for landmine detection was proposed in [8]. It was shown that the proposed approach performed better than the conventional Expectation and Maximisation (EM) HMM on a set of real data. In [9], the authors used an ultrawideband synthetic aperture radar for landmine detection and proposed the use of a sequential floating selection method to reduce the data dimensionality and extract the main features of the landmine. They showed that their method could provide benefits in detection performance against anti-tank landmines. A new learning algorithm based on Gibbs sampling to estimate the parameters of a HMM model with Gaussian mixtures for landmine detection using GPR was presented in [10]. In [11], the authors proposed a set of time-frequency features for the characterisation of ultrawideband signatures of landmines. Full Wave Inversion (FWI) as an imaging tool for GPR was proposed in [12]. The authors investigated if a small antenna array could be used for FWI together with the Singular Value Decomposition to compensate for a lack of multi-offset data and improve the rate of false alarms. The use of GPR 


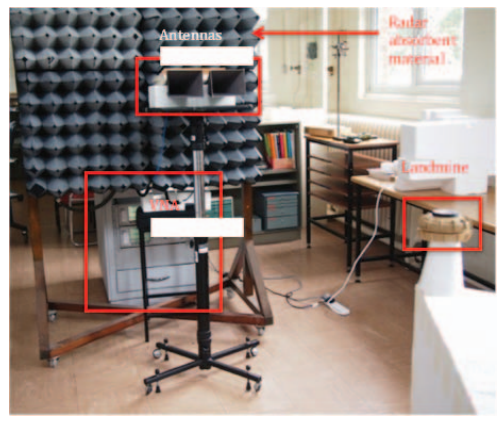

Fig. 1. Experimental set up with the two horns in front of the VNA and facing the landmine under test on the stand. The height of the antennas was $127 \mathrm{~cm}$ and the landmine was placed $205 \mathrm{~cm}$ from the two antenna horns and at height of 135 .

frequency-domain spectral features to improve the detection of weak-scattering plastic mines and to reduce the number of false alarms resulting from clutter was proposed in [13] . In [14], the authors proposed the application of a modern computer vision technique to extract features for landmine detection with GPRs and showed that the technique can provide a robust tool for landmine identification.

The problem with existing vehicle mounted GPRs is that they are large, slow, and require constant supervision from human operators who are inevitably often exposed to unsafe operations. Landmine clearance is a task that is particularly suitable for robotic unmanned platforms as it is repetitive, dangerous and does not need to be carried out with excessive operational speed. Autonomous platforms are becoming a readily affordable technology and funding is currently being spent to facilitate the development of small, light, fast and low cost ultra-wideband GPR systems which can be deployed on unmanned vehicles. When this technology becomes available, it will be possible to survey affected areas with more flexible platform trajectories and this will allow the collection of multi-perspective and potentially multi-static information over much wider angular windows and from many more aspect angles. The use of multi-perspective signatures may allow the exploitation of the behaviour of the landmine signature as a function of the angle of illumination with respect to clutter. Landmines are often symmetrical in shape and hence their signatures may contain features that are less sensitive to the aspect angle compared to those of common cluttered objects. One of the objectives of this project is to study the landmine signatures as a function of the aspect angle, and identify key and robust features that can be used to enhance detection and discrimination performance.

In this paper, we present the results of an experimental trial carried out to collect HRRPs of two landmines, the SB-33 and the VS-50, off the ground. An analysis of the auto-correlation function of each range bin as a function of the aspect angle is also presented together with that of the cross-correlation between profiles collected from different aspect angles.

\section{EXPERIMENTAL SETUP}

The measurements were carried out in June 2014 at the Defence Academy of the United Kingdom. Data was collected with an Anritsu Vector Network Analyser (VNA) transmitting a stepped frequency waveform of a bandwidth $B=3.5 \mathrm{GHz}$,

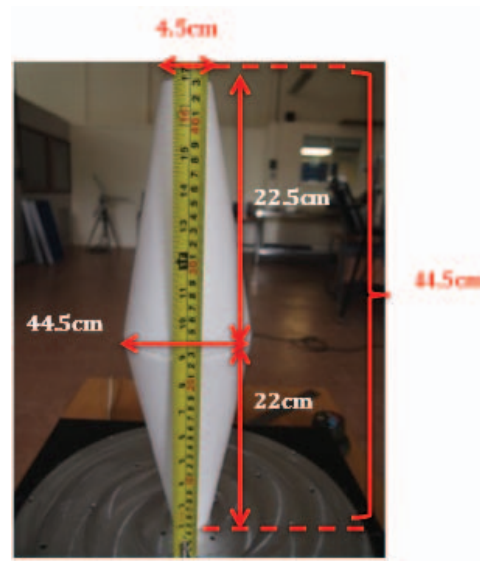

Fig. 2. The stand used to take the measurements consisting of two styrofoam cones on top of each other. The geometry was selected to isolate the landmine returns from the multipath.

\begin{tabular}{|c|c|c|c|}
\hline Type & Diameter & Metal Content & Weight \\
\hline VS-50 & $8.5 \mathrm{~cm}$ & Low (Piston Only) & $185 \mathrm{~g}$ \\
\hline SB-33 & $8.5 \mathrm{~cm}$ & Low (small internal components) & $140 \mathrm{~g}$ \\
\hline
\end{tabular}

from $6.5 \mathrm{GHz}$ to $10 \mathrm{GHz}$, leading to a range resolution of about $4.3 \mathrm{~cm}$. The frequency bandwidth was selected in order to illuminate the landmines with wavelengths comparable to the size of the internal components, despite the compromise with ground penetration which may inevatably limit the use of these frequencies to dry soil or detection of surface landmines. Two identical $15 \mathrm{~cm} \times 11 \mathrm{~cm}$ horn antennas, separated of 4 $\mathrm{cm}$, were used to transmit and receive with the VNA. The landmines under test were placed on a styrofoam cone at the centre of a rotating turntable and measurements were collected with a 10 degree rotation step over 360 degrees. During the measurements the landmines were placed at the same height as the antennas, approximately $1.3 \mathrm{~m}$ from the ground floor, and at a distance of about $2 \mathrm{~m}$ from the radar. A photo of the experimental setup is given in Figure 1. The geometry and the shape of the styrofoam cone, acting as a stand for the target, were carefully selected in order to guarantee that the echo from the target returned to the radar before any multipath reflections resulting from the intereaction between the setup and the target. The styrofoam material was used due to its low reflection properties so to minimising the impact of the stand on the measurements taken. To test the quality of the measurements and ensure multipath free data were collected, a calibrating sphere was firstly placed on the styrofoam cone and measured as it was rotating about the turntable to produce a clean Sinogram. A measurement of the background was collected for each landmine and was subtracted from the data. Data was gathered for two explosive-free anti-personnel landmines, the SB-33 and the VS-50. Photos of both landmines are shown in Figure 3 and Figure 4 and their geometrical characteristics are given in Table I.

\section{RESULTS}

Figure 5 shows the high range resolution profiles for the SB-33 landmine as a function of aspect angle over 360 degrees. The profiles are normalised to the maximum value and are 


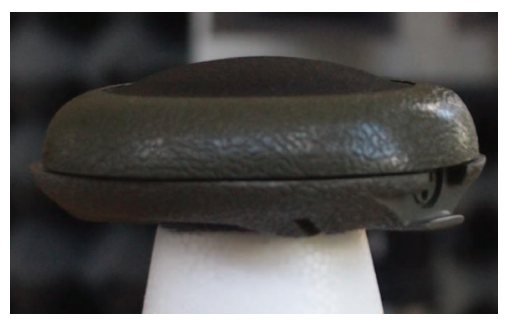

Fig. 3. A photo of the SB-33 landmine.

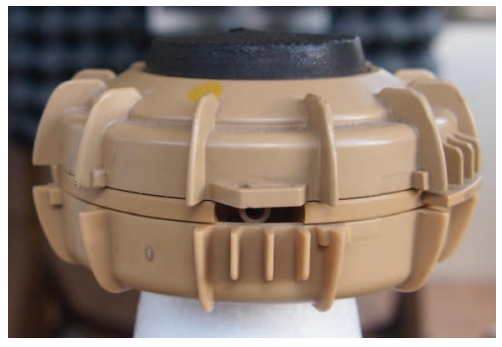

Fig. 4. A photo of the VS-50 landmine.

presented in a dB scale. Results show a main scattering contribution between $205 \mathrm{~cm}$ and $210 \mathrm{~cm}$ which is overall regular with aspect angle. They also show a weaker contribution between $195 \mathrm{~cm}$ and $200 \mathrm{~cm}$ which changes with aspect angle and that may be due to the features of the landmine plastic case.

Figure 6 and Figure 7 show the auto-correlation function of the I and Q components of the signature for each range bin, respectively. This was calculated as

$$
X_{n}(k)=\sum_{i=1}^{N_{p}-k} s_{i}(n) s_{i+k}(n)
$$

where $n$ represents the $n^{\text {th }}$ range bin, $N_{p}$ is total number of range profiles and $s_{i}$ is the I or Q component of the signature at the $i^{\text {th }}$ aspect angle. Results show that there is a high degree of correlation as a function of aspect angle for the range bins between $205 \mathrm{~cm}$ and $215 \mathrm{~cm}$ and that some of this range

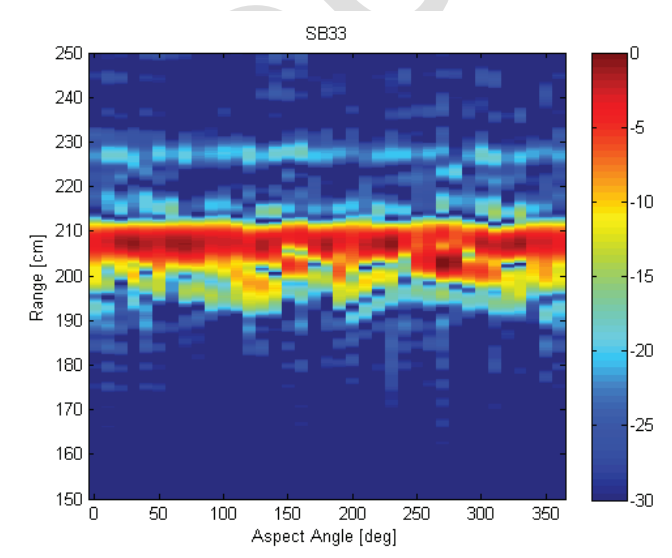

Fig. 5. High Range Resolution Profiles (HRRPs) of the SB-33 landmine as a function of aspect angle over 360 degrees. The image is in a $\mathrm{dB}$ scale and it is normalised to the maximum value.

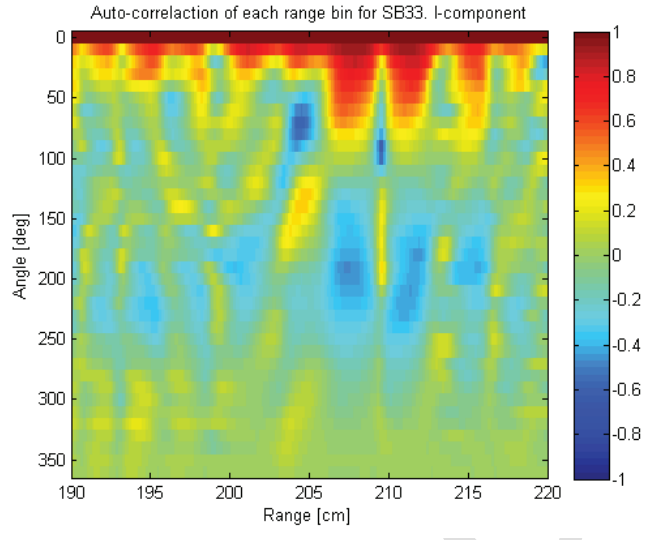

Fig. 6. Autocorrelation function of the in-phase component of each range bin for the SB-33 landmine.

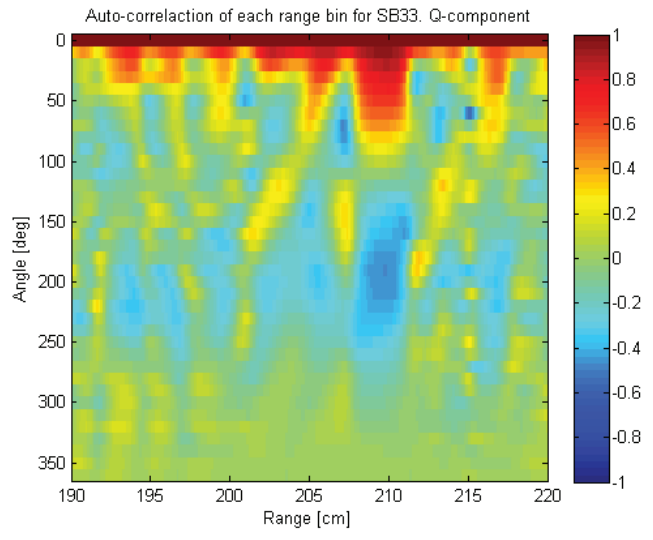

Fig. 7. Autocorrelation function of the quadrature component of each range bin for the SB-33 landmine.

bins can decorrelate with as much as 70 degrees. Figure 8 shows the maximum values of the cross-correlation function between all HRRPs collected from different aspect angles. This was calculated on the zero-mean and unit-power normalised profiles $s_{i}(n)$ as

$$
X_{i j}(k)=\sum_{n} s_{i}(n+k) s_{i+j}(n)
$$

Results show that there are very high-correlation zones for the range profiles taken from aspect angles from 0 degrees to about 100 degrees. On the average results show high levels (over 0.8 ) of cross-correlation between profiles with only very small areas falling below 0.6 cross-correlation.

Figure 9 shows the normalised high range resolution profiles for the VS-50 landmine as a function of aspect angle over 360 degrees. Results show a more regular scattering as a function of aspect angle with respect to the SB-33 landmine. There is a main scattering contribution between $195 \mathrm{~cm}$ and $200 \mathrm{~cm}$ which is stable with respect to aspect angle and another two scattering lines at about $205 \mathrm{~cm}$ and $210 \mathrm{~cm}$ from the radar. Results are in agreement with the more regular shape of this landmine. The scattering contribution is bigger than the landmine itself suggesting the contribution of a stronger multipath interference. Figure 10 and Figure 11 show the auto- 


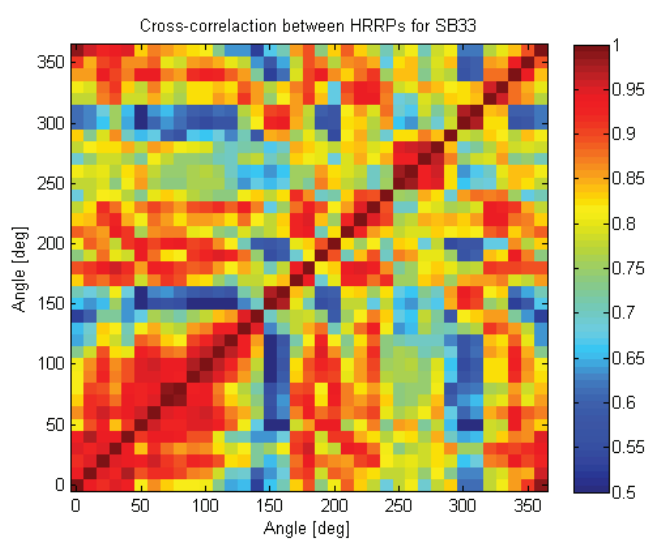

Fig. 8. Maximum values of the cross-correlation between High Range Resolution Profiles of the SB-33 landmine collected from different aspect angles.

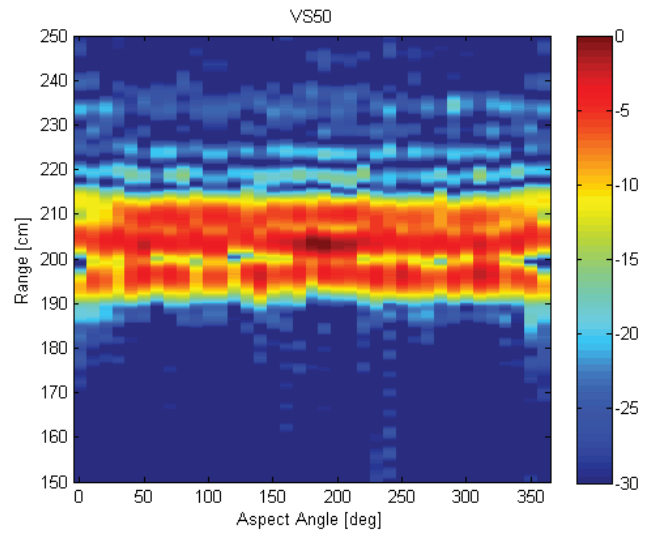

Fig. 9. High Range Resolution Profiles (HRRPs) of the VS-50 landmine as a function of aspect angle over 360 degrees. The image is in a dB scale and it is normalised to the maximum value.

correlation function of the in-phase and quadrature components of each range bin of the VS-50 landmine. As with the SB33 landmine some range bins decorrelate after as much as about 100 degrees and now these extend for a much longer distance from $190 \mathrm{~cm}$ to $215 \mathrm{~cm}$. This is in agreement with the results of the HRRPs that show a much longer response for the VS-50 with respect to the SB-33. Results of the crosscorrelation between range profiles taken from different aspect angles show a much higher degree of correlation with respect to the SB-33, with cross-correlation values being over 0.8 between all profiles. The differences in the level of crosscorrelation between the two landmines (Figure 8 and Figure 12) may be due to the shape of the plastic case of the SB-33 which is not as symmetrical as that of the VS-50.

\section{CONCLUSION}

We carried out an experimental trial to collect multiperspective high range resolution profiles of two landmines off-the-ground and we analysed the cross-correlation properties of the landmine signature as a function of aspect angle. Results showed that, for these measurements, there is a high level of cross correlation between profiles collected from different

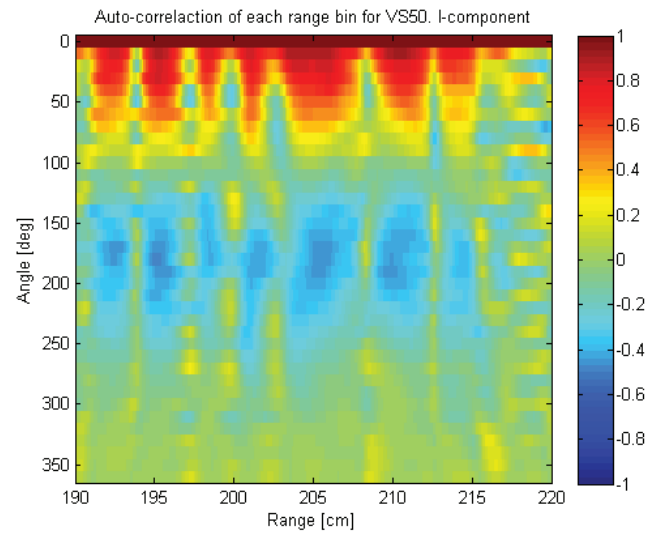

Fig. 10. Autocorrelation function of the in-phase component of each range bin for the VS-50 landmine.

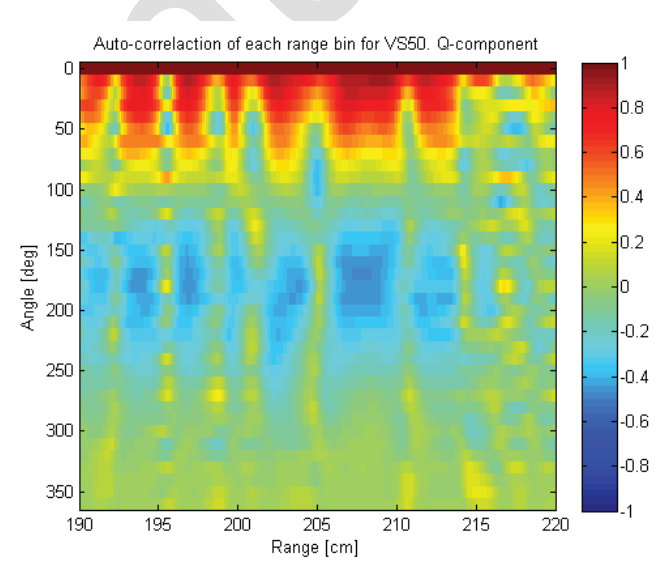

Fig. 11. Autocorrelation function of the quadrature component of each range bin for the VS-50 landmine.

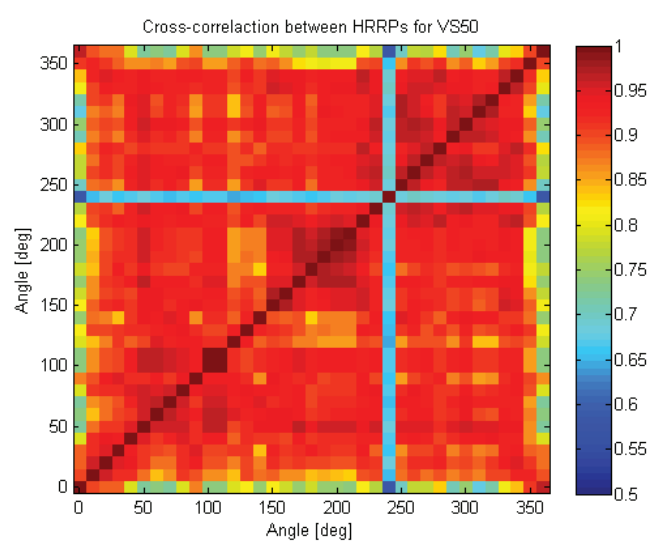

Fig. 12. Maximum values of the cross-correlation between High Range Resolution Profiles of the VS-50 landmine collected from different aspect angles. 
illumination angles. They also showed that the properties of the auto-correlation function of each landmine range bin are such that decorrelation can take place after as much as 70 degrees.

This work has been key to help us develop a working measurement setup but much more work is required at this stage to firstly fully characterise the landmines in free space and then to investigate the effects of different types of soil on the results. The landmine presented in this work were emptied of any explosives but future measurements will be taken with landmines filled with a material with close dielectric properties to those of common explosives. Future work shall look at repeating the measurements at various frequencies and bandwidths, for both landmines and common cluttered objects, with higher angular and range resolution off-the-ground and inthe-ground. This will allow a much more in-depth analysis of the results and the applications of various feature extraction algorithms to the measured signatures, so that an analysis of detection and discrimination performance as a function of aspect angle can be carried out even in more realistic conditions.

\section{ACKNOWLEDGMENT}

The authors thank the Ammunition Hall at the Defence Academy of the United Kingdom for providing the landmines used for the measurements.

\section{REFERENCES}

[1] The Landmine Monitor 2014. International Campaign to Ban Landmines, 2014.

[2] FABW. (May 2015) Find a better way. [Online]. Available: http://www.findabetterway.org.uk/landmine-facts/

[3] D. Daniels, Ground Penetrating Radar. Institution of Electrical Engineers, London, 2004.

[4] A. Langman, M. R. Inggs, and B. C. Flores, "Improving the resolution of a stepped frequency cw ground-penetrating radar,' Proceedings of SPIE, vol. 2275, pp. 146-155, 1994.

[5] A. Langman and M. R. Inggs, "Electromagnetic model for a steppedfrequency continuous-wave ground-penetrating radar," Proceedings of SPIE, vol. 4758, pp. 291-296, 2002.

[6] A. Langman and M. Inggs, "The use of polarimetry in subsurface radar," in Geoscience and Remote Sensing Symposium, 1994. IGARSS '94. Surface and Atmospheric Remote Sensing: Technologies, Data Analysis and Interpretation., International, vol. 4, Aug 1994, pp. 2489-2491 vol.4.

[7] A. Langman and M. R. Inggs, "Polarimetric model for a steppedfrequency continuous-wave ground-penetrating radar," Proceedings of SPIE, vol. 4758, pp. 645-651, 2002.

[8] A. Manandhar, P. Torrione, L. Collins, and K. Morton, "Multipleinstance Hidden Markov Model for GPR-based landmine detection,' IEEE Transactions on Geoscience and Remote Sensing, vol. 53, no. 4, pp. 1737-1745, April 2015.

[9] T. Jin and Z. Zhou, "Ultrawideband synthetic aperture radar landmine detection," IEEE Transactions on Geoscience and Remote Sensing, vol. 45, no. 11, pp. 3561-3573, Nov 2007.

[10] X. Zhang, J. Bolton, and P. Gader, "A new learning method for continuous hidden markov models for subsurface landmine detection in ground penetrating radar," IEEE Journal of Selected Topics in Applied Earth Observations and Remote Sensing, vol. 7, no. 3, pp. 813-819, March 2014.

[11] T. Savelyev, L. van Kempen, H. Sahli, J. Sachs, and M. Sato, "Investigation of time ndash;frequency features for gpr landmine discrimination," IEEE Transactions on Geoscience and Remote Sensing, vol. 45, no. 1, pp. 118-129, Jan 2007.
[12] F. Watson and W. Lionheart, "SVD analysis of GPR full-wave inversion," in 15th International Conference on Ground Penetrating Radar (GPR), 2014, June 2014, pp. 484-490.

[13] K. Ho, L. Carin, P. Gader, and J. Wilson, "An investigation of using the spectral characteristics from ground penetrating radar for landmine/clutter discrimination," IEEE Transactions on Geoscience and Remote Sensing, vol. 46, no. 4, pp. 1177-1191, April 2008.

[14] P. Torrione, K. Morton, R. Sakaguchi, and L. Collins, "Histograms of oriented gradients for landmine detection in ground-penetrating radar data," IEEE Transactions on Geoscience and Remote Sensing, vol. 52, no. 3, pp. 1539-1550, March 2014. 